teins was antagonised by simultaneous injection of mannose terminal glycoproteins and vice versa, suggesting that there are unlikely to be independent Kupffer cell receptors responsible for clearance of mannose or $\mathrm{N}$-acetylglucosamine-terminal glycoproteins respectively. Presumably the Kupffer cell receptor has relaxed specificity and binds to mannose residues present in both glycoprotein types. It has recently been suggested that the hepatocyte receptor also does not discriminate between galactose and glucose terminals in some circumstances (Stowell et al. Fedn Proc. 36, 653; 1977). In this property the mammalian receptors resemble the plant lectins which in general also bind to several different sugars. Since both forms of $\beta$-glucuronidase are efficiently cleared from the circulation, the Kupffer recognition system does not require a phosphorylated site on the lysosomal enzyme, raising fascinating speculation on the potential role of phosphorylation in

\section{Diffusion in polymers}

\section{from Paul Calvert}

Semicrystallinf, polymers behave as if they consisted of two phases, crystal and amorphous, arranged in alternate layers. Most simple properties are a function of the fractional crystallinity but are insensitive to the structure or arrangement of the phases. Some recent measurements on the diffusion of chain molecules in polyethylene serve as a reminder that diffusion can tell us about structural details.

According to the two-phase model a semicrystalline polymer consists of plate-like crystals of about $10 \mathrm{~nm}$ thickness separated by amorphous regions of the same size. The crystals have the same properties as hypothetical large crystals and the amorphous material should be the same as completely amorphous polymer. This idea works well for bulk properties such as density, modulus, heat capacity and expansion coefficient (see Wunderlich Macromolecular Physics I, Academic Press, 1973). There are probably crystal defects and special crystal surface structure but they do not affect bulk properties.

It seems reasonable that motion in the amorphous phase will be limited by the crystals, but in polymers such as polystyrene and polyethylene terephthalate 1 am not aware of any evidence for a change in glass transition temperature or mobility between com-

Paul Calvert is in the School of Molecular Sciences at the University of Sussex. deciding the circulatory fatc and target cell of glycoproteins.

In summary, therefore, the original findings of Ashwell and Morell have opened up to reveal a remarkable collection of cellular receptors for glycoproteins. These receptors differ in specificity according to cell type, for example the preference of the mammalian hepatocyte receptor for galactosyl terminals, the Kupffer and fibroblast cell receptors for mannosyl residues and phosphomannosyl residues respectively while the identification of the avian hepatic receptor as a binding activity for $\mathrm{N}$-acetylglucosaminyl terminated glycoproteins (Lunney \& Ashwell Proc. natn. Acad. Sci. U.S.A. 73, 341 ; 1976) warns against extrapolation across species. The list almost certainly will continue to grow and underlines the feeling that carbohydrate structure is involved in a variety of recognition systems, and defects in such recognition may correlate with some human disease states.

pletely amorphous and partly crystalline polymers. Boyer has suggested that this happens in polyethylene (Macromolecules 6, 288; 1976) but the situation is still confused. Intercrystalline links are chains running from one crystal to another stitching the structure together (Keith \& Padden J. appl. Phys. 42, 4585; 1971). These should be more frequent in quickly cooled samples where the crystals are small and have proved useful in explaining effects of molecular weight and cooling rate on mechanical properties. Unfortunately, no very convincing way of detecting them in normal samples has been found (although see Wunderlich Makromol. Chem. 175, 977; 1974). So, although strength and deformation behaviour do depend on structural factors other than crystallinity, and although likely factors have been discussed, there is not much supporting evidence. Diffusion measurements could be very informative about this.

Many measurements of diffusion of gases in polymers were made in the 1950 s and early 1960 s when plastic packaging was becoming important (see Crank \& Park Diffusion in Polymers Academic Press, 1968; Rogers in Physics and Chemistry of the Organic Solid State 2, Interscience 1965). It is probably these workers whom we should thank for the disappearance of the soggy potato crisp. In semicrystalline polymers it was found that gas solubilities were proportional to the amorphous fraction, implying that gases are excluded from the crystals. Michaels and coworkers (J. Polymer Sci., 41, 53; 1959; 50, 393, 413; 1961) described diffusion by $D=D_{\mathrm{a}} / \tau \beta$ where $D_{a}$ is the diffusion coefficient in pure amorphous material, $\tau$ is a 'tortuosity factor' dependent on crystallinity and $\beta$ is an immobilisation factor which is dependent on the size of the diffusing molecule. They initially found that in branched and Ziegler polyethylenes $\tau$ was proportional to amorphous content to a power between -1 and -2 rather than the expected inverse proportionality. In later measurements on linear polycthylcne (J. appl. Phys. 35, 3165; 1964) they did find diffusion to be simply proportional to amorphous content in samples crystallised by cooling. By annealing, however, they could produce samples of the same crystallinity but with different diffusion rates. This topic has essentially remained in this state since.

Klein and Briscoe made measurements of the diffusion of long chain amides in polyethylene (Nature 257, 386; 1975; Polymer 17, 481; 1976), and have recently extended this to study morphological effects on the diffusion of chain molecules (Nature 266, 43; 1977; J. Polymer Sci. (Phys.) (in the press)). They cnvisage the diffusion of a chain molecule in the interlamellar regions as the motion of a large snake across the floor of a forest, winding amongst the intercrystalline links. This is based on the ideas of de Gennes (J. Chem. Phys. 55, 572; 1971; I. de Physique 36, 1199; 1975), and monte carlo calculations for two dimensional snake-like diffusion ('reptation') around obstacles by Doi (J. Phys. A 8, 417; 1975). With some rather sweeping assumptions about the frequency of intercrystalline links they are able to calculate relative diffusion rates for two compounds containing 45 and 25 carbon atoms in rapidly and slowly cooled samples. The calculated ratios are in respectable agreement with experiment. The calculated and observed diffusion rates are faster in the more crystalline, slowly cooled, sample in contradiction to the results of Michaels for gases, and to simple crystallinity theories.

At the moment this is a lot of theory based on little data, and there are many unanswered questions including why annealed samples are different from continuously cooled ones, why Ehy found more rapid diffusion in film surfaces (J.appl. Phys. 35, 2720; 1964) and whether diffusion is homogeneous throughout the spherulites. Nonetheless, a quantitative theory is much better than a qualitative model and we should lcarn a great deal about morphology in proving it right or wrong. $\square$ 